East African Medical Journal Vol. 86. No. 12 December 2009

PREVALENCE OF ADVERSE DRUG REACTIONS IN ADULT PATIENTS ON ANTI-RETROVIRALS AT KENYATTA NATIONAL HOSPITAL-COMPREHENSIVE CARE CENTRE

L.E.M. Mwangangi, MPharm, Tutorial Fellow, Department of Pharmaceutics and Pharmacy Practice, College of Health Sciences, University of Nairobi, P.O. Box 19676-00202, Nairobi, Kenya, R. Juma, MBChB, MMed, Centre for Clinical Research, Kenya Medical Research Institute, P.O. Box 19463- 00202, Nairobi, Kenya, D.K. Scott, PhD, Associate Professor, D.G.Nyamu, MPharm, Tutorial Fellow and K.A.MKuria, PhD, Senior Lecturer, and Chairman, Department of Pharmaceutics and Pharmacy Practice, College of Health Sciences, University of Nairobi, P.O. Box 19676-00202, Nairobi, Kenya

Request for reprints to: Dr. L.E.M. Mwangangi, Department of Pharmaceutics and Pharmacy Practice, College of Health Sciences, University of Nairobi, P.O. Box 19676-00202, Nairobi, Kenya

\title{
PREVALENCE OF ADVERSE DRUG REACTIONS IN ADULT PATIENTS ON ANTI-RETROVIRALS AT KENYATTA NATIONAL HOSPITAL, COMPREHENSIVE CARE CENTRE
}

\author{
L.E.M. MWANGANGI, R. JUMA, D.K. SCOTT, D.G. NYAMU and K.A.M. KURIA
}

\begin{abstract}
Background: There has been an increased access to anti-retrovirals in resourceconstrained settings. However, few studies have addressed the area of adverse drug reactions in these settings.

Objective: To determine the prevalence of adverse drugs reactions in HIV-infected persons receiving anti-retrovirals.

Design: A retrospective cohort study.

Setting: Kenyatta National Hospital, Comprehensive Care Centre.

Subjects: Adult patients receiving anti-retroviral drug during the four year period between January 2003 and December 2006.

Main outcome measures: The primary outcome was a documented adverse drug reaction after exposure to antiretrovirals.

Results: Systematic random sampling was used to pick 350 patients' files. There were 219 recorded adverse drug reactions in $170(48.6 \%)$ patients (some patients had more than one adverse drug reaction). Peripheral neuropathy was the most common adverse drug reaction with a prevalence of $28.9 \%$ followed by lipid abnormalities at $14.3 \%$. Conclusion: This study indicates a high prevalence of adverse drug reactions among HIV/AIDS patients on anti-retroviral therapy at Kenyatta National Hospital, Comprehensive Care Centre.
\end{abstract}

\section{INTRODUCTION}

There has been an increased international will and commitment to expand programmes for providing highly active anti-retroviral therapy (HAART) to those who need them. These include the ' $3 \times 5$ ' initiative by the World Health Organisation (WHO), the Global fund for AIDS, TB and malaria, and the U.S. government's presidential emergency plan for AIDS relief (PEPFAR). All these have resulted in increased access to HAART for HIV infected individuals in resource-constrained settings. However, few studies have addressed the area of adverse drug reactions (ADRs) in these settings $(1,2)$.

Most of the published studies have been from developed countries which have advanced ADRs monitoring and reporting systems. In Kenyatta National Hospital (KNH), as in most other parts of the country, the emphasis has been on trying to put as many eligible patients as possible on HAART. Being a resource constrained facility, there have been few ADR studies.

Monitoring of ADRs is particularly important for anti-retroviral drugs since many are licensed on the basis of limited safety data and they are likely to be used in combinations untested in pre-marketing studies.

Further, individuals with HIV infection are often treated with many other drugs for the prevention and treatment of opportunisticinfections and there are a number of potential interactions with anti-retroviral drugs (3).

In developed countries, liver-function tests, and serum creatinine and lipid levels are routinely used in assessing patients for ADRs (1). However, countries with limited resources lack the laboratory infrastructure to perform these assays routinely. 
With increased access to HAART, it is important that developing countries should undertake further clinical research to optimise guidelines for the care of HIV-infected patients in local situations. In an effort to address the above needs, a consensus conference was held to discuss priorities for anti-retroviral therapy research in Zambia, one of the world's most heavily HIV-afflicted nations. There was global consensus that anti-retroviral clinical trials in resource-constrained countries were possible, and the capacity for such trials should be developed further in Africa (4).

The World Health Organisation defines an adverse drug reaction (ADR) as "A response to a drug which is noxious and unintended, and which occurs at doses normally used in humans for prophylaxis, diagnosis or therapy of disease, or for the modification of physiological function" $(5,6)$. This definition excludes therapeutic failures, intentional and accidental poisoning and drug abuse. Using this conservative definition avoids overestimating the ADR prevalence.

All anti-retroviral drugs have both short-term and long-term ADRs. The risk of specific ADRs varies according to specific drug, class of drug and individual susceptibility.

A better understanding of the ADRs of antiretroviral agents is of interest to optimise therapy in HIV infected patients (7).

\section{MATERIALS AND METHODS}

This hospital based retrospective cohort study was carried out at the Kenyatta National Hospital (KNH) Comprehensive Care Centre (CCC).

Data collection and analyses: Around 4000 patients were initiated on HAART between January, 2003 and December, 2006. The records were arranged in order of HAART initiation date and every fifth file was examined for possible inclusion. Of the 831 files examined, 350 met the inclusion criteria for the study. These were all adult patients ( $\geq 18$ years at the time of diagnosis) with HIV / AIDS initiated into HAART at the CCC in KNH during the four year study period whose baseline laboratory results were available in the files and whose baseline laboratory results and initial clinical examination did not indicate a preexisting ADR related condition.

The sampled files were reviewed to pick out clinician records of any of the specified six ADRs, patient characteristics and baseline laboratory tests. Relevant data were entered into a pre-designed data collection form before transfer into the Statistical Package for Social Sciences (SPSS 13.0) database for analysis.

Case definitions: The primary outcome was having a documented ADR after exposure to HAART.
The cases were defined according to the guidelines contained in the Kenya National Clinical Manual for ART providers (8), which mainly follows the criteria used by the AIDS Clinical Trials Group (ACTG) (9). Peripheralneuropathy (PN). Patients were considered to have experienced peripheral neuropathy if they presented with any of the symptoms ranging from mild tingling sensation, numbness, muscle weakness and pain to severe incapacitating pain and inability to walk (8).

Lipid abnormalities: Lipid abnormalities (LA) were recorded if the patient had a hump on the back, loss of facial fat, thinning of arms or central fat deposition (8). Serum lipid profiles were not used in defining lipid abnormalities because records of lipid profiles for both before and after the initiation into HAART were not available for most files.

Hepatoxicity: A patient was recorded as having hepatoxicity if the levels of alanine aminotransferase (ALT) rose above $40 \mathrm{IU} / \mathrm{L}$ (9). Severe liver toxicity (grade 3 or 4) was defined as a greater than five fold increase in levels of ALT and/or aspartate aminotransferase (AST) above the upper limit of normal (ULN), following the criteria used by the AIDS Clinical Trials Group (ACTG) (9).

Haematological toxicity: Patients were considered to have haematological toxicity if the level of haemoglobin $(\mathrm{Hb})$ was less than $8 \mathrm{~g} / \mathrm{dl}$ or neutrophils less than 1000 $/ \mathrm{mm}^{3}$ after exposure to the drugs (8).

Skin rash: Patients were considered to have skin rashes if there were records of the patient having skin conditions ranging from mild cases of erythema and pruritus to severe mucous membrane involvement (e.g., Stevens-Johnson syndrome) (8).

Renal abnormalities: Renal abnormalities were recorded if the renal function tests showed rising levels of serum creatinine above $150 \mu \mathrm{mol} / 1$ after exposure to the drugs (8).

Data quality control: Before the full study, the investigator consulted the primary clinicians involved in the direct patient care at the $\mathrm{KNH}, \mathrm{CCC}$ to understand how they documented ADRs and the terms which they used to describe the ADRs in the CCC. This helped in the design of an appropriate data collection form. The reliability of the data were enhanced by having a second person (peer) to fill a 'data reliability form' by randomly sampling one out of every ten files studied by the investigator. The data reliability form was exactly the same as the data extraction form except that it was filled by a different person. The two were then compared and any discrepancies addressed. 


\section{RESULTS}

A total of 831 files were retrieved from the $\mathrm{KNH}$, CCC records. Four hundred and eighty one (57.9\%) of the retrieved files were excluded because: $34.5 \%$ had been on HAART for less than a year, $8.4 \%$ were patients who had started HAART elsewhere before the median time on HAART was 32 (range 12-56) months.

Age and gender distribution of the sample: The majority of patients $(79.4 \%)$ were in the 31-50 year age group. There was a higher proportion of males aged above 40 years $(57 \%)$ compared to the females $(40.4 \%)$ above

Figure 1

Age and gender distribution of the study population

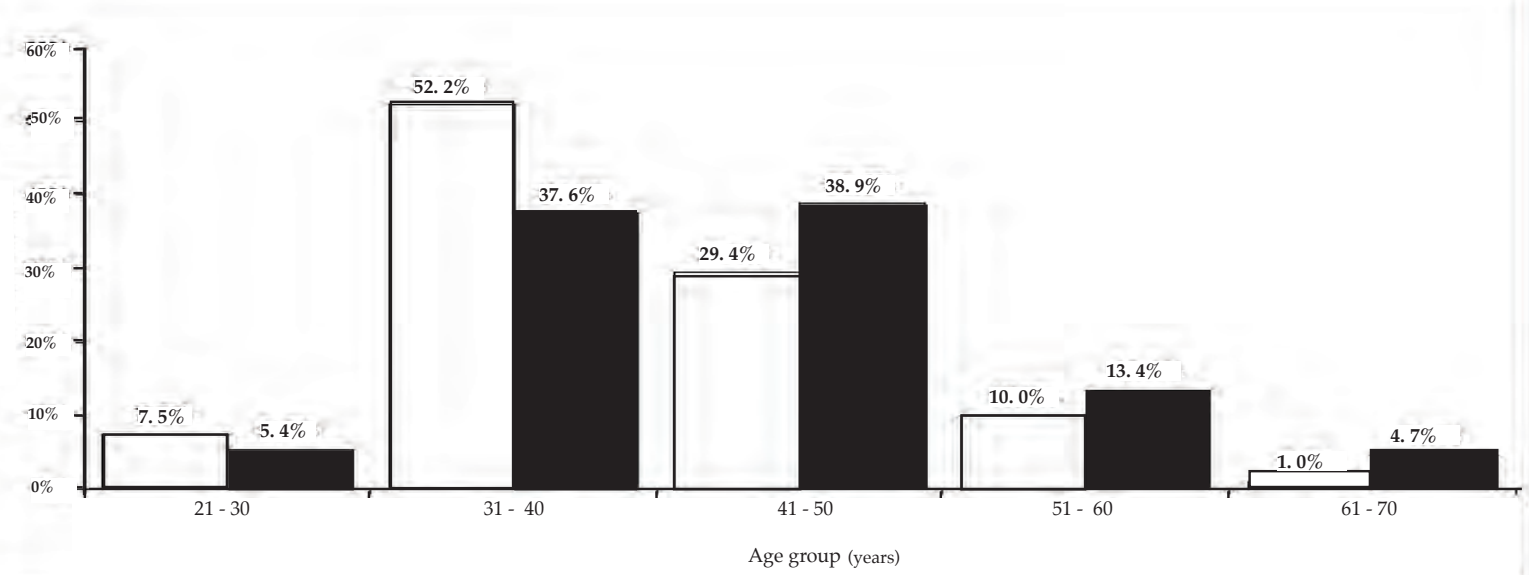

transferring into $\mathrm{KNH}$, hence had no baseline records, $7.3 \%$ were below 18 years of age, $4.7 \%$ date of starting HAART was not indicated, 1.8\% had incomplete HAART records and $1.1 \%$ started HAART before January 2003. The remaining 350 patients were enrolled into the study.

The study participants had a median age of 40 (range 23 - 65) years, 57\% were female, 52\% were married and $91 \%$ had some formal education. The median CD4 count was 123 (range 1 - 904) while
40 years. Males were generally older than the females (median age 42 vs 39, $\mathrm{p}=0.003$ ) (Figure 1).

HAART regimens dispensed: All patients on treatment were started on protease inhibitor-sparing regimens with $92 \%$ on Stavudine (D4T) based regimens. The most common regimen was the stavudine, lamivudine and nevirapine combination which accounted for $56 \%$ of the regimens used (Figure 2).

\section{Figure 2}

Categories of HAART regimens among study population prevalence of ADRs

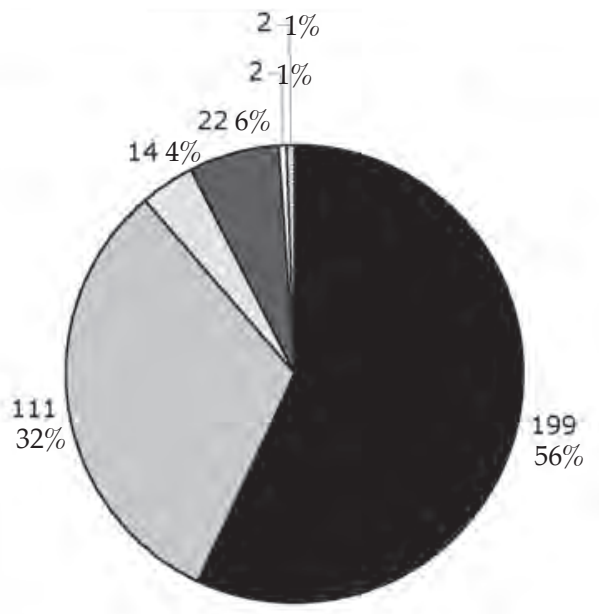


Figure 3

Prevalence of ADRs in the study participants

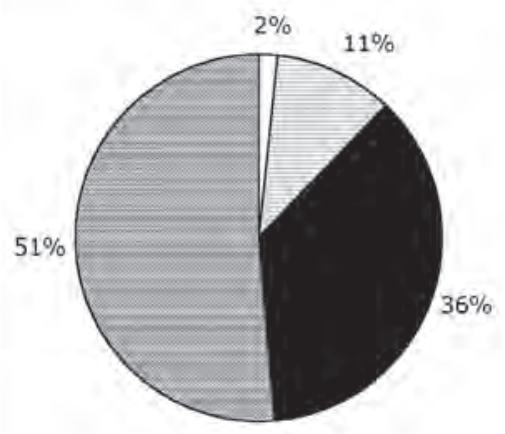

Prevalence of specific ADRs: Peripheral neuropathy was the most common ADR with a prevalence of $28.9 \%$ followed by lipid abnormalities at $14.3 \%$. (Figure 4).

Time to development of first ADR: The median time to development of first ADR ranged from two months for rashes to 29 months for lipid abnormalities. First cases of most ADRs were seen early except for renal and lipid abnormalies whose first cases occurred in the second year of HAART (Table 3). Rashes and renal abnormalities had a narrow range. A wide range was seen with the other ADRs (Table 1).

Trends/Distribution of ADRs over time: Prevalence of all the ADRs generally increased with time on HAART. The prevalence of at least one ADR increased from $29 \%$ in the first 12 months to $58 \%$ by the $48^{\text {th }}$ month. There seemed to be a plateau phase after 48 months on HAART (Figure 5).

Figure 4

Prevalence of specific ADRs in the study participants

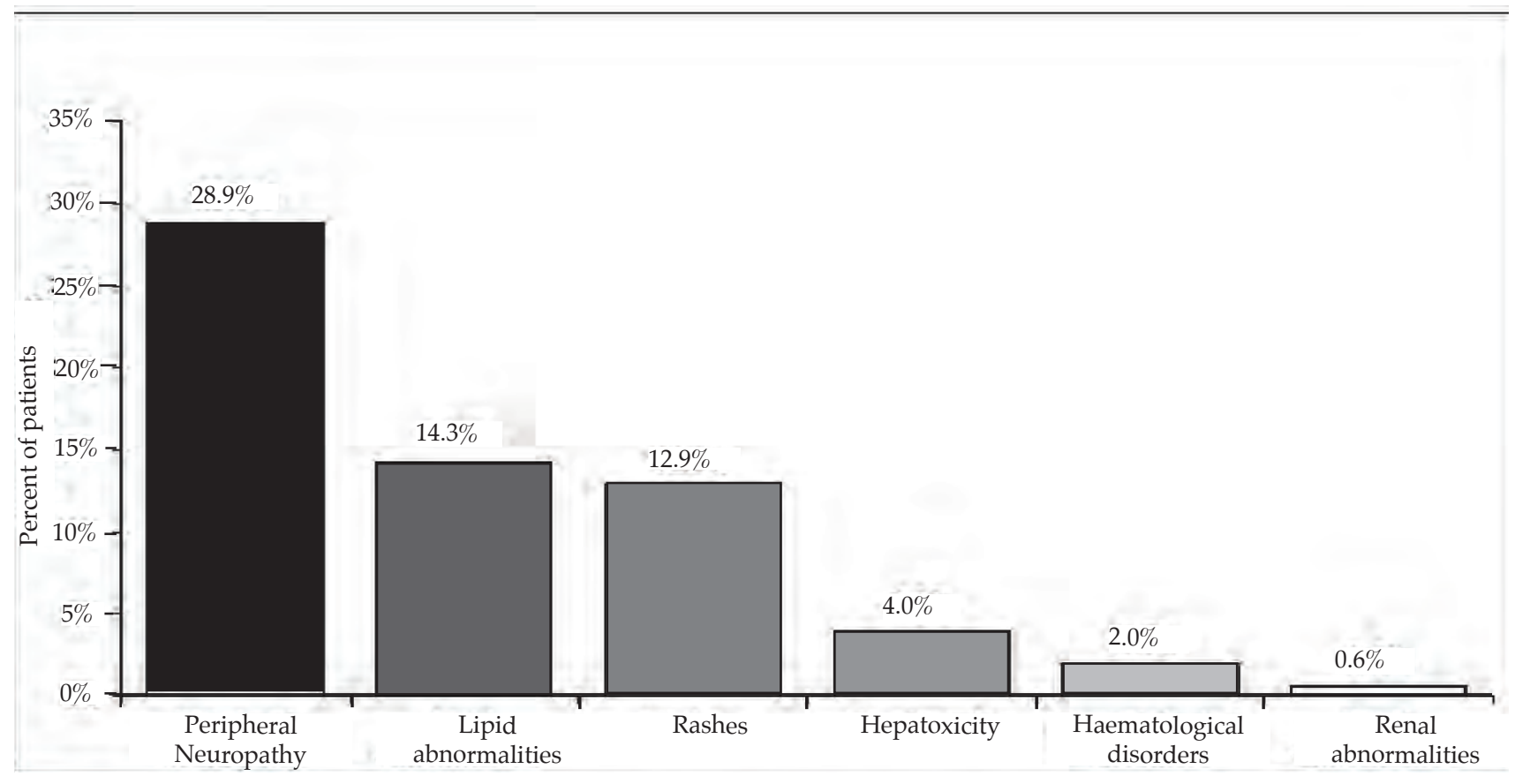

Table 1

Time (in months) to development of first ADR

\begin{tabular}{lcccccc}
\hline Rashes & $\begin{array}{c}\text { Haematological } \\
\text { disorders }\end{array}$ & $\begin{array}{c}\text { Peripheral } \\
\text { neuropathy }\end{array}$ & Hepatoxicity & $\begin{array}{c}\text { Renal } \\
\text { abnormalities }\end{array}$ & $\begin{array}{c}\text { Lipid } \\
\text { abnormalities }\end{array}$ \\
\hline Median & 2 & 4 & 8 & 11.5 & 18.5 & 29 \\
Range & $<1-7$ & $<1-39$ & $<1-49$ & $<1-33$ & $17-20$ & $11-50$ \\
\hline
\end{tabular}


Figure 5

Prevalence of ADRs with increasing time on HAART (categorised in 12 month intervals)

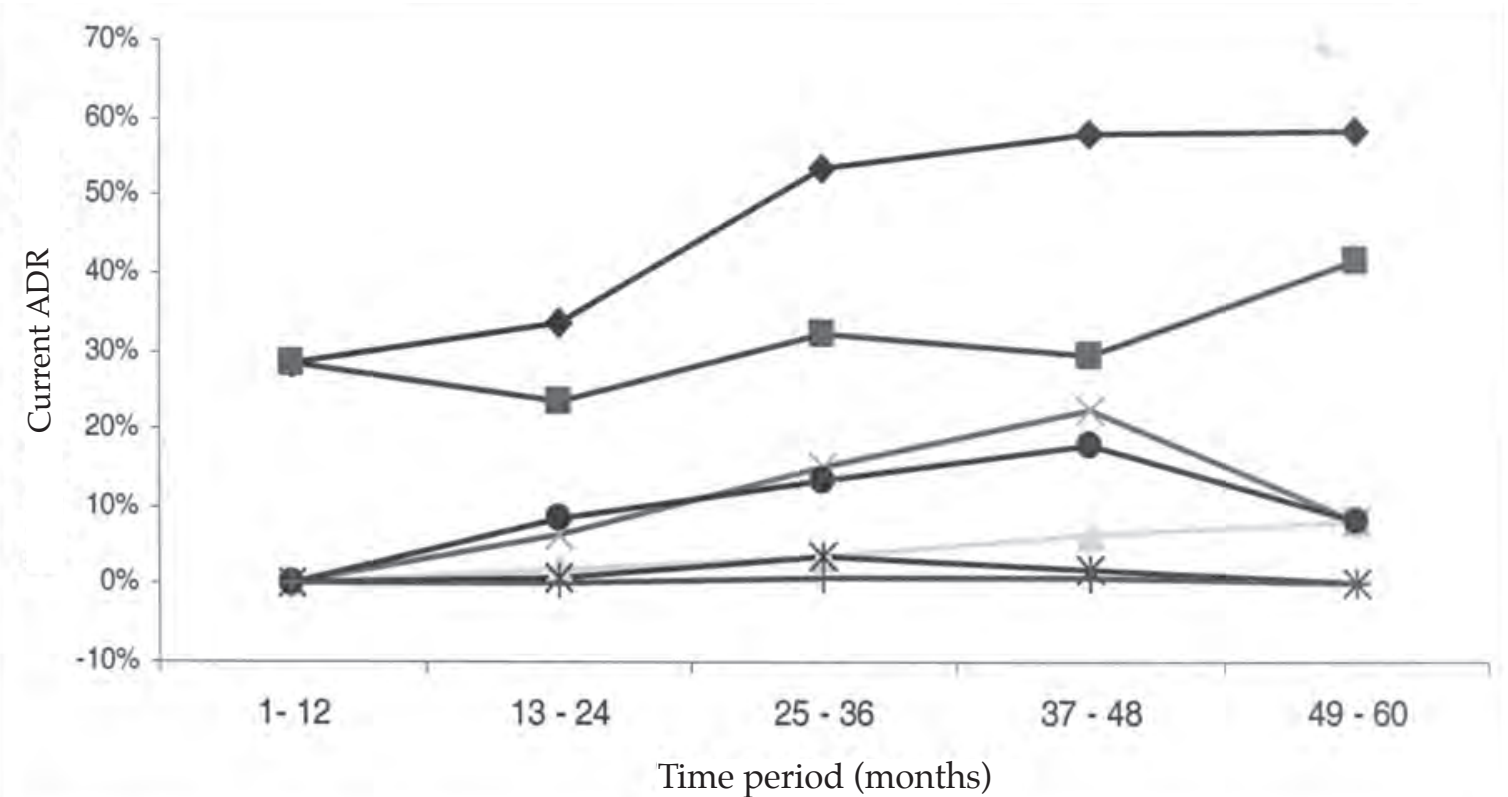

\begin{tabular}{|c|c|c|}
\hline —ーoverall_ADR & hy - hepatoxicity & $\rightarrow$-lipid_abnormalities \\
\hline *-haematological_disorders $\longrightarrow$ rashes & —renal_abnormalities & \\
\hline
\end{tabular}

\section{DISCUSSION}

The current study is one of the few ADR studies carried out in resource-limited settings. The results suggest a high prevalence of ADRs among patients on HAART and hence need for their closer monitoring and management.

Of concern in this study is that 287 patient files perused $(34.5 \%)$ had to beexcluded because the patients stayed on HAART for less than a year. The high drop out from the programme in the first year needs to be investigated as some of these withdrawals may be due to intolerable ADRs or disease related deaths.

In this cohort females were more $(57 \%)$ and relatively younger than their male counterparts (median age of 39 vs 42 and mean 40 vs $43, p=0.004$ ). Fifty seven per cent of males were aged above 40 years compared to $40.4 \%$ females. This compares well with the national estimates that there are more of younger HIV-infected women than men in Kenya (10).

The most common regimen was stavudine, lamivudine plus nevirapine. This is in keeping with the national guidelines to use this regimen as the standard first line HAART (8). The high (95\%) compliance to the national guidelines on HAART initiation serves to demonstrate the potential of well written guidelines to standardise pharmaceutical care of complex disease conditions like HIV / AIDS in resource-limited settings.
Forty nine per cent of patients developed at least one ADR in this cohort; previous studies have reported antiretroviral toxicity in $11-76 \%$ of their cohorts, but comparison between studies is difficult as a result of differences in reporting of ADRs; studies may report overall ADRs, severe ADRs only, treatment-limiting ADRs, or laboratory diagnosed ADRs only (11-13). Nevertheless, this prevalence of ADRs compares well with other studies in resource-limited settings. For example, in an observational study carried out between 2003 and 2004 in rural Uganda on 1029 adult HIV / AIDS patients on HAART, ADRs were found in $40 \%$ of the patients (14). This study was similar to our study in that it reported clinically apparent ADRs only.

Our results compare well with those obtained in a prospective study conducted from 2001 to 2003 to assess factors associated with ADRs among individuals initiating HAART at two public referral HIV/AIDS centres in Brazil which reported occurrence of at least one ADR in $34.5 \%$ of the 397 patients although they did include patients who had been on HAART for less than one year (12).

However, our results differed slightly from those obtained in a study carried out in St. Mary's Mission Hospitalin Nairobi, from September 2004 until August 2006 (15). The study which involved a total of 1286 patients reported that HAART related clinical toxicities occurred in $26.5 \%$ of patients. Though the two studies 
were conducted in the same town, the difference in results could be due to the different methodology, duration ofstudy and population characteristics. While the St Mary's study was a longitudinal observation of clinical, immunologic and treatment parameters of all HIV-infected adult patients initiated on HAART with at least one follow-up visit, the KNH study was retrospective and included only those patients who had been on HAART for at least a year. Moreover, the St Mary's study covered two year duration unlike our study which followed patients for up to five years. The longer follow up in our study may have contributed to the higher prevalence observed as a result of accumulation of ADRs with time.

The six ADRs of interest in our study differed markedly in their prevalence and time of occurrence. This suggests that clinicians should look out carefully for ADRs in each and every visit to avoid more harm occurring to the patients.

The prevalence of peripheral neuropathy was $28.9 \%$ in our study making it the most common ADR. This was consistent with findings from several other studies in resource-limited settings. In the two studies carried out at rural Uganda and St Mary's hospital in Nairobi, peripheral neuropathy was reported most frequently with a prevalence of $36 \%$ and $20.7 \%$ respectively $(14,15)$. Peripheral neuropathy was also the most common ADR in a cross-sectional study involving 720 patients in a large teaching hospital in Malawi (16).

Lipid abnormalities were the second most common ADR with a prevalence of $14.3 \%$. This compares well with a combined retrospective and prospective study conducted on 83 adult HIVtreatment-naïve patients in Thailand (17) which reported that $16.8 \%$ had symptoms of lipodystrophy within two years of treatment with a combination of stavudine, lamivudine plus nevirapine, the most common HAART regimen in our study.

However, the results from our study are different from those obtained in 409 patients starting WHO recommended first-line HAART regimens in Rwanda which reported a high prevalence of lipodystrophy of $34 \%$ (18). Whilst ours was a purely retrospective study, the Rwanda study subjected the patients to a standardised case definition-based questionnaire and clinical assessment. This may have made it much easier to pick ADRs. The Rwanda study also had a high proportion of patients who had been on HAART for longer than our patients. It has been observed that the risks of lipid abnormalities increase with time on HAART.

A cross-sectional study at $\mathrm{KNH}$ between January and April 2006 reported a high prevalence of dyslipidemia of $63.1 \%$ (19). This study differs from the current study in that it involved doing lipid profiles for all patients unlike in the current study which recorded clinically apparent lipid abnormalities only. This highlights the need for routine laboratory monitoring of all the patients at regular intervals in order to detect any abnormalities before they are clinically apparent.

The prevalence of rashes in our cohort was low (12.9\%); in contrast, a prospective study on 202 patients in Thailand reported rash in $34 \%$ of patients on nevirapine or efavirenz (20). This is not surprising because a reduced occurrence of rashes has been noted in Africa for other medications like cotrimoxazole (21). Our findings are similar to those obtained in several other studies in Africa; the study in rural Uganda also reported a low frequency of rash $(6 \%)$ (14) while rashes occurred in approximately $3.7 \%$ of patients in the St Mary's (Kenya) study (15). This may partly be explained by the fact that it is much harder to see rashes on the dark skin of Africans compared to the lighter skin of Asians.

Hepatoxicity associated with nevirapine use has been reported with varying frequency in many studies of HAART-related toxicity in resource-limited settings (22). However, though several antiretrovirals have been reported to cause fatal acute hepatitis, most often they only cause an asymptomatic elevation of ALT (23). In our study, hepatoxicity occurred in approximately $4 \%$ of patients, which is higher ratethan among several cohorts in resource-limited settings. The Uganda and Kenya studies reported a frequency of acute hepatitis of $0.5 \%$ and $0 \%$ respectively $(14,15)$. The difference could be because these two studies reported only acute cases. The difference may also be attributed to the low use of laboratory diagnosis of hepatoxicity and other ADRs in resource-limited settings. However, our results compare well with those of a retrospective study involving 3154 patients at a tertiary HIV care referral centre in South India which reported hepatitis in 3.5\% of patients (24).

The prevalence of haematological disorders of $2 \%$ in our study compares well with other studies in resource-limited settings; a prospective cohort study on 169 patients in Cameroon found that anaemia was rare with a prevalence rate of $4 \%$ and $6 \%$ for zidovudine and stavudine containing regimens respectively (25). The study in South India reported that clinically significant anaemia was observed in $5.4 \%$ of patients (25), while the study in rural Uganda reported an even lower frequency of $0.4 \%$ (15).

The findings in this study that the occurrence of lipid abnormalities differed markedly from that of rashes in that, while the first cases of rashes were reported within a few days after HAART initiation, no cases of lipid abnormalities were seen before eleve months of HAART. This suggests the need for frequent and early monitoring for rashes while the late occurrence of lipid abnormalities suggests that clinicians should watch out for them on all patients who have been on treatment for duration of about one year or more. 
Our study was limited by potential of either over or under-reporting of ADRs in patient files. This being a retrospective study, it relied on pre-recorded information which may have been incomplete or inaccurate. Also, most of the recorded ADRs were those which were clinically apparent. ADRs that required laboratory diagnosis may have been missed because tests were not done routinely due to cost limitations.

In conclusion, this study indicates a fairly high prevalence of ADRs among HIV/AIDS patients on HAART at Kenyatta National Hospital Comprehensive Care Centre. This being among the first few studies on ADRs of antiretrovirals in Kenya, further research is required. This is important because of the rapidly expanding access to antiretrovirals, the lack of regular laboratory monitoring in these settings and the wide range of ADR prevalence reported in the few studies done so far.

\section{ACKNOWLEDGEMENTS}

To the management and staff of the Kenyatta National Hospital for their contribution to this study.

\section{REFERENCES}

1. Reynolds, S. J., Bartlett, J.G., Quinn, T.C., et al. Antiretroviral therapy where resources are limited. N. Engl. J. Med. 2003; 348: 1806 - 1809.

2. Wanchu, A., Pareek S., Bambery P., et al. Adverse drug reactions to generic antiretroviral medication in resource constrained settings. Conf. Retrovir. Opportunistic Infect. 2006; 13: 562.

3. Hooker, M., Arlett, P.R, Lee, G.H., et al. HIV adverse drug reaction reporting scheme. Int. Conf. AIDS.1998; 12: 90. Abstract no. 12385.

4. Zulu, I., Schuman, P., Musonda, R., et al. Priorities for antiretroviral therapy research in sub-Saharan Africa:2002Consensus ConferenceinZambia. J. Acquir. Immune. Defic. Syndr. 2004; 36: 831-834.

5. van den Bemt, P.M., Egberts T.C., de Jong-van den Berg, L.T. and Brouwers, J.R. Drug-related problems in hospitalised patients. Drug. Saf. 2000; 22: 321-333.

6. World Health Organization. International Drug Monitoring: The role of the hospital. Geneva, Switzerland: World Hlth. Org. 1966. Technical Report Series No. 425.

7. Bates, D.B., Leape, L.L. and Petrycki, S. Incidence and preventability of adverse drug events in hospitalised adults. J. Gen. Intern. Med. 1993; 8: 289-294.

8. Kenya National Clinical Manual for ART Providers, Ministry of Health, $2^{\text {nd }}$ Edition, 2007.

9. AIDS Clinical Trials Group 1996. Table of grading severity of adult adverse drug experiences. US Division of AIDS, National Institute of Allergy and Infectious Diseases, Bethesda, Rockville, MD.

10. Ministry of Health. AIDS in Kenya: Trends, Interventions and Impact. 2005, $7^{\text {th }}$ edition. 7-14.
11. Laurent, C., Kouanfack, C., Koulla-Shiro, S., et al. Effectiveness and safety of a generic fixed-dose combination of nevirapine, stavudine, and lamivudine in HIV-1-infected adults in Cameroon: open-label multicentre trial. Lancet. 2004; 364: 29-34.

12. Pádua, C.A., César, C.C., Bonolo, P.F., Acurcio, F.A. and Guimarães, M.D. High incidence of adverse reactions to initial antiretroviral therapy in Brazil Braz. J. Med. Biol. Res. 2006; 39: 495-505.

13. Wester, C.W., Kim, S., Bussmann, H., et al. Initial response to highly active antiretroviral therapy in HIV-1C-infected adults in a public sector treatment program in Botswana. J. Acquir. Immune Defic. Syndr. 2005; 40: 336-343.

14. Forna, F., Liechty, C.A., Solberg, P., et al. Clinical toxicity of highly active antiretroviral therapy in a home-based AIDS care program in rural Uganda. Acquir. Immune. Defic. Syndr. 2007; 44: 456-462.

15. Hawkins, C., Achenbach, C., Fryda, W., et al. Antiretroviral durability and tolerability in HIVinfected adults living in urban Kenya. J. Acquir. Immune. Defic. Syndr. 2007; 45: 304-310.

16. van Oosterhout, J.J., Bodasing, N., Kumwenda, J.J., et al. Evaluation of antiretroviral therapy results in a resource-poor setting in Blantyre, Malawi. Trop. Med. Int. Hlth. 2005; 10: 464-470.

17. Tin, E.E., Bowonwatanuwong, C., Desakorn, V., et al. The efficacy and adverse effects of GPO-VIR (stavudine+lamivudine+nevirapine) in treatmentnaïve adult HIV patients. Southeast Asian J. Trop. Med. Pub. Hlth. 2005; 36: 362-369.

18. van Griensven, J., De Naeyer, L., Mushi, T., et al. High prevalence of lipoatrophy among patients on stavudine-containing first-line antiretroviral therapy regimens in Rwanda. Trans. $R$. Soc. Trop. Med. Hyg. 2007; 101: 793-798.

19. Manuthu, E.M., Joshi, M.D., Lule, G.N. and Karari, E. Prevalence of dyslipidemia and dysglycemia in HIV infected patients. East Afr. Med. J. 2008; 85: 10-17.

20. Ananworanich, J., Moor, Z., Siangphoe, U., et al. Incidence and risk factors for rash in Thai patients randomized to regimens with nevirapine, efavirenz or both drugs. AIDS. 2005; 19: 185-192.

21. Mermin, J., Lule, J., Ekwaru, J.P., et al. Effect of cotrimoxazole prophylaxis on morbidity, mortality, CD4-cell count, and viral load in HIV infection in rural Uganda. Lancet. 2004; 364: 1428-1434.

22. Sanne, I., Mommeja-Marin, H., Hinkle, J., et al. Severe hepatoxicity associated with nevirapine use in HIV infected subjects. J. Infect. Dis. 2005; 191: 825-829.

23. Núñez, M. and Soriano,V. Hepatoxicity of antiretrovirals: incidence, mechanisms and management. Drug. Saf. 2005; 28: 53-66.

24. Kumarasamy, N., Venkatesh, K.K., Cecelia, A. J., et al. Spectrum of adverse events after generic HAART in Southern Indian HIV-infected patients. AIDS. Patient Care STDS. 2008; 22: 337-344.

25. Laurent, C., Bourgeois, A., Mpoudi-Ngolé, E., et al. Tolerability and effectiveness of first-line regimens combining nevirapine and lamivudine plus zidovudine or stavudine in Cameroon. AIDS Res. Hum. Retroviruses. 2008; 24: 393-400. 